\title{
NEUTRAL-NEUTRAL REACTIONS: A NEW CRESU STUDY
}

\author{
C. Rebrion, A. Defrance, J.L. Queffelec, B.R. Rowe and D. Travers, \\ Département de Physique Atomique et Moléculaire \\ Université de Rennes 1 \\ 35042 RENNES Cedex \\ FRANCE
}

\begin{abstract}
A new CRESU experiment devoted to neutral-neutral chemistry at low temperature is being built . The first reactions to be studied are $\mathrm{N}+\mathrm{NO}$ and $\mathrm{CN}+\mathrm{O}_{2}$.
\end{abstract}

\section{Introduction}

Models of interstellar clouds usually emphasize the importance of ion chemistry in spite of the fact that recent theoretical studies [1] [2] have stressed out that many neutral reactions may have no barrier on the potential surface. The dynamics of such reactions would be dominated by long-range forces and in some cases the reaction could be very fast.

In a comparison of two current models, Millar et al. [3] have shown that the calculated abundances of several molecules depend critically on the choice of the rate coefficient for some neutral reactions. However, models have to rely on functional forms chosen either for lack of better information or from theoretical guidance. Even at liquid nitrogen temperature there is a scarcity of experimental data concerning gas phase neutral chemistry [4] and no data at temperatures relevant for interstellar chemistry.

\section{The experiment}

The CRESU method, based on the supersonic expansion of a buffer gas through a Laval nozzle, is now well known in the astrochemical community. The first version, located in Meudon (France), is devoted to ion-molecule reactions at very low temperature. As ions make very easily clusters and also because of termolecular reactions, a very low pressure is needed. Such problems are much less important in neutral chemistry and higher pressure in the flow can be used. The DPAM is building a new CRESU experiment working in a higher pressure range which will be used to study neutral chemistry down to $10 \mathrm{~K}$.

Two glass nozzles are available: one helium nozzle working at $\mathrm{T}=140 \mathrm{~K}$, and one nitrogen nozzle working at $\mathrm{T}=170 \mathrm{~K}$ (lower temperatures can be reached when the reservoir of the nozzles is cooled with liquid nitrogen: $T=37 \mathrm{~K}$ and $\mathrm{T}=45 \mathrm{~K}$ respectively). Their characteristics have been tested using two independent methods (impact pressure measurements and time of flight measurements) and both sets of results are in good agreement. 
3. $\mathbf{N}+\mathrm{NO} \rightarrow \mathbf{N}_{2}+\mathrm{O}$

The data available in the $160-400 \mathrm{~K}$ range agree to find its rate coefficient temperature independent. However, theoretical calculations by Jaffe et al. [5] show that an energy barrier of several meV could occur on the reaction path, which should induce a reactivity variation at $45 \mathrm{~K}$.

The cooled nitrogen nozzle will be used for this study. A mixture of $\mathrm{N}_{2}$ and NO will be injected through the nozzle and a micro-wave discharge will dissociate $\mathrm{N}_{2}$ into $N\left({ }^{4} S\right), N\left({ }^{2} D\right)$ and $\mathrm{N}\left({ }^{2} \mathrm{P}\right)$. The population of the metastable states of $\mathrm{N}$ will be checked, in order to take into account the deexcitation processes and the different reaction rates with NO.

\section{4. $\mathrm{CN}(\mathrm{v}=0)+\mathrm{O}_{2} \rightarrow \mathrm{NCO}+\mathrm{O}$}

The sketch of the experiment needed for this study in drawn below. The reaction will be studied with the helium nozzle. The $\mathrm{CN}$ radical is obtained by laser photolysis of NOCN (synthetized itself from $\mathrm{NO}, \mathrm{Cl}_{2}$ and $\mathrm{AgCN}$ ) at $532 \mathrm{~nm}$. Its decay will be followed by laser induced fluorescence tuned to a rotational line in the $\mathrm{B}-\mathrm{X}(0,0)$ band.

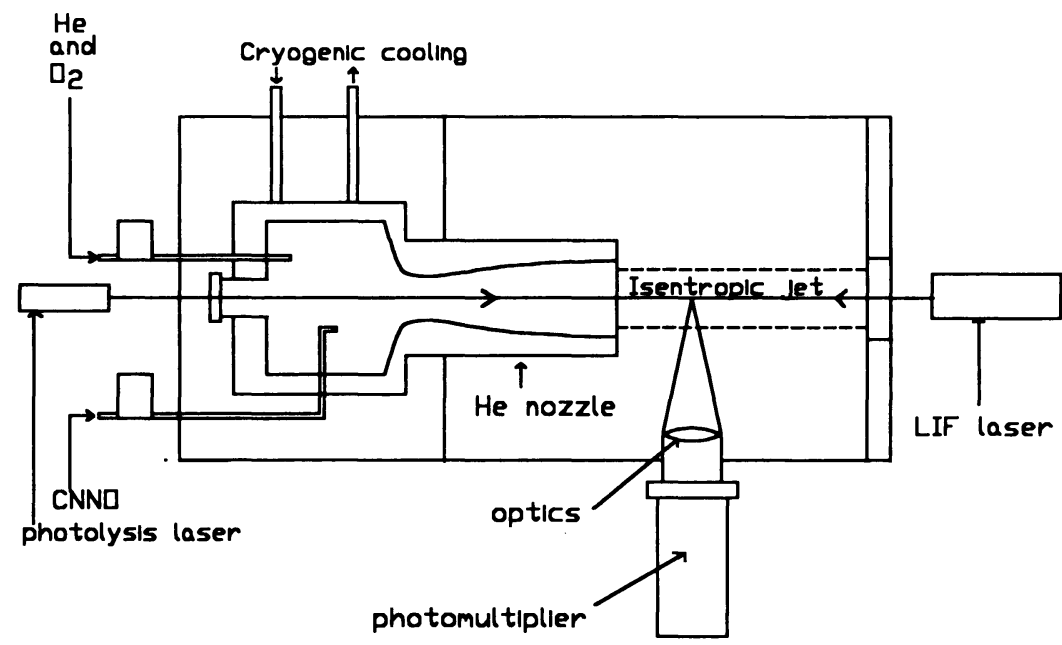

[1] D.C. Clary , Mol. Phys. $\underline{53}, 3,1984$

[2]M.M. Graff, Astrophys. J. 339, 239, 1989

[3] T.J. Millar, C.M. Leung, E. Herbst, Astron. Astrophys. 183, 109, 1987

[4]M.J. Frost, P. Sharkey, I.W.M. Smith, Faraday Discuss. Chem. Soc. 1991, in press

[5] S.P. Walch, R.L. Jaffe, J. Chem. Phys. 6, 6946, 1987 